\title{
Mechanisms of Dermatophyte Invasion on New Zealand Rabbit Skin Models
}

\author{
Faisal Jamin ${ }^{1}$, Sri Estuningsih ${ }^{2}$, Eko S.Pribadi ${ }^{2}$, and Ekowati Handharyani2 ${ }^{2}$ \\ ${ }^{1}$ Faculty of Veterinary Medicine, Universitas Syiah Kuala, Jln. Tgk. Hasan Krueng Kalee No. 4 Kopelma Darussalam, Banda \\ Aceh, Indonesia, 23111 \\ ${ }^{2}$ Faculty of Veterinary Medicine, Bogor Agricultural University, Jln. Agatis, Kampus IPB Dramaga Bogor, Kabupaten Bogor, \\ Indonesia, 16680
}

\begin{abstract}
Dermatophytosis is a dermatophyte fungi infection most commonly found in animals and humans. The first step of infection is started from the attachment of arthroconidium into stratum corneum. The attachment of fungi to the host cell is mediated by fungi adhesin and its interaction with the host receptor. The objective of this research was to develop the infection model of Microsporum gypseum and Microsporum canis by inoculating the macroconidia intradermally on the rabbit model. The macroconidia collection method from culture media was re-visualized as fungi ultrastructure using scanning electron microscopy (SEM). Skin lesion analysis was measured from clinical changes of the skin based on primary dermatophytosis signs such as circular alopecia with erythema and squamosal. Clinical confirmation test was done via skin sampling followed by histopathological examination using Methenamine Silver-Grocott's (GMS) staining. As a result of this research, the in vivo infection model through direct infection of macroconidia applied intradermally was very effective in improving the direct infection to the invasion phase on the skin. This model confirmed the epidermal differentiation process and skin permeability showed primary lesion within 2 hours and aggravated up to 6 hours after inoculation. In conclusion, macroconidium is a potential source of infection to induce the dermatophytosis model and the severity of primary injection correlated with duration and the scale of clinical symptoms exhibited. This is a promising model for further research on the mechanism involved in dermatophyte infection.
\end{abstract}

Keywords: dermatophyte, dermatophytosis, macroconidia, rabbit model.

\section{Introduction}

Dermatophytosis or tinea is a skin disease caused by dermatophyte fungi that can infect a cat, dog, cow, sheep, goat, horse, camel, monkey, rabbit, experimental animals, and human. Dermatophyte is the most common cause of fungal infection in the world [1]. Dermatophyte fungi within the genus microsporum, trichophyton, and epidermophyton cause various infections in the skin keratin layer. Based on its habitat, these fungi are classified as anthropophilic, zoophilic, and geophilic. Dermatophyte fungi which are keratolytic cause damage to the skin (epidermis and dermis), hair, and nail $[2,3]$. Dermatophytosis cases often found in pet and domesticated animals, and can even infect humans. Indonesia is located in a tropical region with high humidity, a suitable place for growth for several types of fungi [4-6]. Dermatophytosis in Bali cow has been reported by (7), causing economic loss to farmers. [8] stated that more than USD 500.000.000 per year is spent for dermatophytosis treatment in the world. Thus, there is a need for continuous research development on dermatophytosis.
The host and fungi relationship aspects, including the invasion mechanism, are still unclear. Existing literature on the dermatophyte invasion mechanism still cannot provide a consistent model, especially regarding experimental and clinical data in treating dermatophytosis. The existing model still showed varieties of clinical infection replicating in animals or humans. Development of a dermatophyte infection model in an animal is among the ways to obtain different dermatophytosis model that is stable for research. Every fungal species has a different colonization ability and microscopic morphology with the tendency for a certain location of the infection. Moreover, different species may cause varied spectrum width of clinical manifestation depending on the degree of severity on the host. In the previous study, spontaneous recovery in the model animal that has been inoculated by conidia by stratum corneum often happens. This makes retaining the dermatophytosis model condition difficult for keratinocyte induced immune response against dermatophyte $[9,10]$.

Elevation of fungi infection is influenced by adhesion time and the number of conidia attached, be it in vitro or in vivo. Dermatophyte growth is followed by the germination phase and then the invasion phase into

* Corresponding author: ekowatieko@apps.ipb.ac.id 
stratum corneum by penetrating fungi element and enzyme secretion which can degrade skin structure [11]. Zurita and Hay [12] stated that an in vitro test utilizing a stratum corneum biopsy layer, it was known that Trichophyton sp. macroconidia can attach to human keratinocyte within 3-4 hours. Aljabre, Richardson [13] stated that $T$. mentagrosphytes macroconidia attachment happened in 6 hours after contact with the host cell and germination occurred in 4 hours after inoculation. The growing hyphae will be identifiable after the next 10 hours. Another experiment performed by Kaufman, Horwitz [11] in the ex vivo model utilizing human skin epidermis explant able to show that attachment and germination process respectively happen after 12 hours and 24 hours. Penetration process into stratum corneum occurred in 3 days post-inoculation (p.i.).

Intradermal macroconidia inoculation allows antigen deposition. Macroconidia is a saprophytic conidium of stable dermatophyte fungi on damaged hair or soil [7]. This stability allows macroconidia to function as the source of infection potential as an antigen to present the dermatophytosis model for the development of medicine and medicine application test against the disease. Macroconidia morphology is very important in classifying and identifying dermatophytes [14]. The dermatophyte invasion mechanism is still unclear, whether in vitro or in vivo model. This research aimed to obtain a more stable dermatophytosis model for research purposes.

\section{Materials and Methods}

\subsection{Time and Location of Study}

The research was performed in the Research Laboratory of Animal Hospital (Rumah Sakit Hewan PendidikanRSHP), Medical Microbiology Division Department of Animal Disease and Veterinary Public Health, and Pathology Division Department of Clinic Reproduction and Pathology, Faculty of Veterinary Medicine, IPB university. Fifteen healthy male New Zealand White rabbits free off dermatophytosis weighing on average 1.5 $\mathrm{kg}$ obtained were obtained from Livestock Research Institut (Balai Penelitian Ternak-BALITNAK) Bogor, Bogor regency, Indonesia. All experiments in this research have been approved by the animal ethics committee of IPB University (No 123-2018 IPB, August 2018).

\subsection{Dermatophytes culture}

Microsporum gypseum and Microsporum canis are two fungi obtained from dermatophytosis clinical samples from dogs and cats in the Bogor region. The dermatophytes were grown in Sabouraud Dextrose Agar (SDA) medium. The cultures were incubated in $\pm 26^{\circ} \mathrm{C}$ temperature and observed every day for 2 weeks. Every isolate was inoculated in 3 petri dishes. Culture is an important accessory in all systemic infection treatment. Identifying characteristics include colony pigment, texture, and growth, morphological structure, microconidia, macroconidia, spiral, pectinate branch, pedicels, and nodular organs [5].

\subsection{Ultrastructure finding of dermatophytes culture}

Dermatophyte specimens were washed 3 times with cacodylate buffer and followed by glutaradehyde on $4^{\circ} \mathrm{C}$ temperature. Samples and then dehydrated with graduated alcohol (50-95\%), and then transferred to be dried in $a$ freeze drier/vacuum drier until dried and then layered with $\mathrm{Au}$ by ion coater tool. Interaction between dermatophyte fungi was observed under the JSM-5310LV electron microscope (Jeol, Jepang). SEM was used to observe the visualization of ultrastructure and to take the image of dermatophyte $[15,16]$.

\subsection{Preparation and standardization of dermatophytes}

Macroconidia collection was collected from SDA media incubated at $26^{\circ} \mathrm{C}$ temperature for 2 weeks. Dermatophyte growth in petri dish was observed every day. Macroconidia concentration was determined by hemocytometer and adjusted to $3 \times 10^{6} \mathrm{~mL}$. Macroconidia suspension was taken from the grounded petri dish colony and centrifuged in peptol water suspension, which was resuspended with distilled water to obtain an even clearer suspension. Macroconidia was analyzed by Olympus optical CO microscope (400x magnification), Ltda BX40 (Tokyo, Japan) [17, 18]

\subsection{Infection and sensitization of animals}

Two dermatophyte strains were inoculated intradermally to the skin by insulin syringe in two different animal model group to obtain the right clinical symptoms describing primary infection. Fifteen rabbits were divided into 3 treatment groups. Rabbits were inoculated by macroconidia on day 0 in the order of group $1(M$. gypseum) and group 2 (M. canis); while the rabbits of the negative control group were not infected but injected with distilled water with a method modified from [19-21]. Data were taken from day 1 up to day 20 post-infection (p.i.). Before inoculation, every rabbit was anesthetized via intramuscular route by the combination of Ketamine (40 $\mathrm{mg} / \mathrm{Kg} \mathrm{BW})$ and Xylazine $(10 \mathrm{mg} / \mathrm{Kg} \mathrm{BW})$. The hair on the back of the model rabbit was cut and shaved in $5 \times 8$ $\mathrm{cm}^{2}$ wide. The remaining hair was abraded gently with a sterile scalpel. Macroconidia suspension in $3 \times 10^{6} / \mathrm{mL}$ concentration contained in distilled water was carefully injected intradermally on six sites by the abraded skin region with a method modified from [22]. For success validation of the animal model, skin and hair scrape from inoculation site were directly observed in $\mathrm{KOH} 10 \%$ for microscopic hyphae examination. Skin reaction was observed in 15 minutes, 30 minutes, and 6 hours postinfection (p.i.). Lesion observation was performed with 24 hours interval for 20 days. Lesions measured were edema, erythema, and skin inflammatory reactivity index [23]. Post-infection (p.i) observation of the skin was followed by skin samples collection which showed a lesion on day 
10 and day 20. The skin samples were obtained through biopsy utilizing a $10 \mathrm{~mm}$ dermal punch, which was then processed for histopathology preparations of the infection site. Skin sample collection wounds were treated by applying betadine and administered ketoconazole $(0.2$ $\mathrm{mg} / \mathrm{cm}^{2}$ ) once a day. Treatment was continued to day 10 and day 20 post skin biopsy. Animal model infected by $M$. gypseum and $M$. canis were nursed by normal saline compresses. The negative control group that is not inoculated was being treated by normal saline compresses with the same volume. Observation of intradermal macroconidia inoculation in animal model rabbit includes mycology examination against hyphae and conidia; clinical evaluation, and histopathological examination. Histopathological observation of dermatophytosis used GMS and HE staining. The observation was performed by Olympus optical CO microscope (400x magnification), Ltda BX40 (Tokyo, Japan).

\section{Results and Discussion}

M. gypseum dermatophyte obtained from cat clinical isolate and M. canis obtained from dog clinical isolate were managed to be grown in SDA medium, from which macroconidia of said dermatophytes were obtained. Macroconidia from dermatophyte are important as an infectious agent. Macroconidia obtained from the culture were used as the infection material in this invasion research. The macroconidia examination includes SEM visualization. Morphology identification examination is discussed more specifically in other topics. Inoculation of M. gypseum and M. canis in rabbit model caused lesion in $100 \%$ of the animal model. The lesion started to be seen on day 1 up to day 3 p.i. signified by inflammation in the form of edema and erythema on the skin [23]. Circular alopecia and skin desquamation started to appear on day 3 and day 4 p.1. Skin lesion finally formed dermatophytosis characteristics sign of circular lesion with the edges being reddish skin inflammation and the center having thinner reddish skin. This lesion is known as ringworm lesion [24]. The diameter measurement of the ringworm zone formed on day 10 was $5.5 \mathrm{~mm}$ and day 20 was $14.5 \mathrm{~mm}$ (Figure 1). Part of skin covered by the white-yellowish layer attached very strongly to the epidermis and progressively develop along with the duration of the infection. Dermatophytosis model success validation results in this research used skin and hair scrapes from inoculation location which was directly examined with $\mathrm{KOH} 10 \%$, where hyphae branching and arthrospores were found [23].
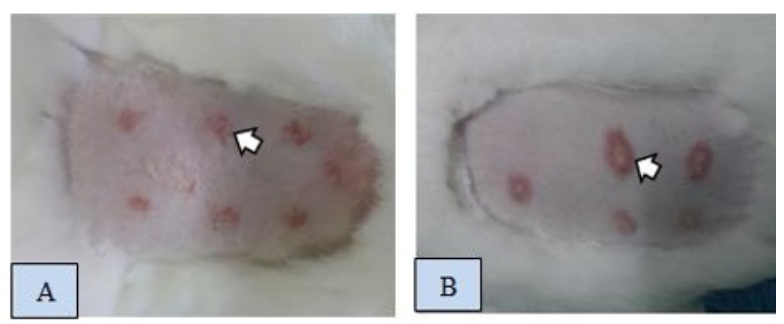

Fig. 1. Clinical lesions of dermatophytosis after infection day 10 of dermatophytes ((arrows); A. M. gypseum; B. M. canis.
Skin histopathology examination of ringworm zone with HE staining showed an inflammatory reaction on the papillary dermis, acanthosis dan hyperkeratosis (Figure 2A, 2B, 2C, and 2D). Hyphae colony of both $M$. gypseum and $M$. canis could be seen in stratum corneum. Hyphae colony of $M$. gypseum appeared larger compared to $M$. canis colony and both were located in stratum corneum, appearing clearly with GMS staining (Figure 2E and $2 \mathrm{~F}$ ). The infected group showed invasion on the epidermis layer and moved to the surface, breaking through keratinocyte and stratum corneum layer - a trait that fits the characteristic dermatophytosis pathology. HE staining showed hyperkeratosis and acanthosis. Fungi presence is hard to examine with HE staining and only showed the damage to the skin tissue structure. Methenamine Silver Grocott's (GMS) staining revealed many conidia colored black with different sizes and septate hyphae all over the epidermis.
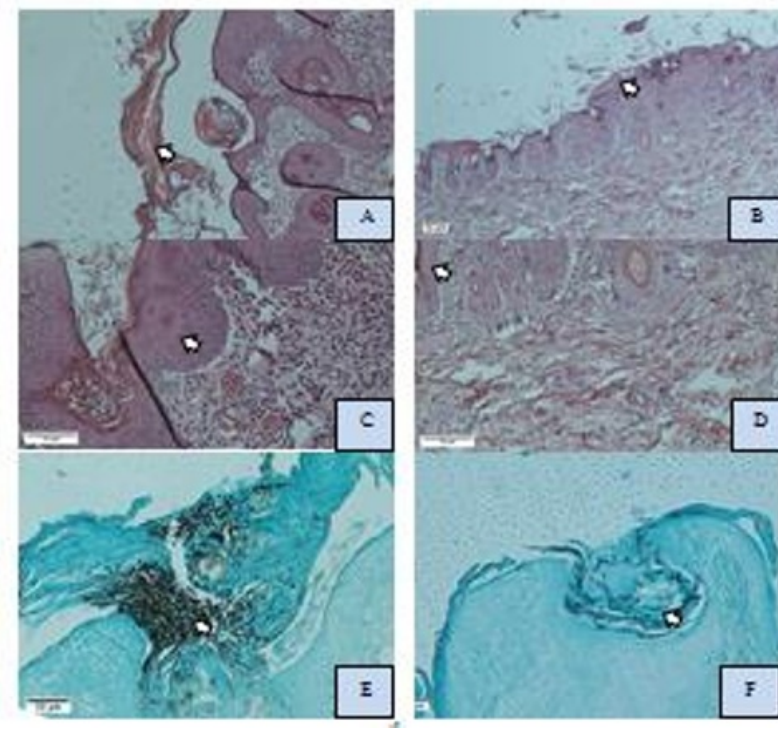

Fig. 2 Histopathology of $10^{\text {th }}$ day inoculated rabbit skin. A, C, E; M. gypseum. B, D, F; M. canis. A, B, C, D; hyperkeratosis and acanthosis (arrows) staining of H\&E. E and F; hyphae and conidia (arrows) staining of GMS

The right model to simulate dermatophyte infection on hosts is a requirement in studying the dermatophytosis infection mechanism. [25] In their in vivo research stated that dermatophyte infection is only limited to stratum corneum. The number of conidia during infection showed the further process of adhesion and stratum corneum invasion to be intuitionistic after infecting 400 conidia. This model imitates natural $T$. rubrum infection, where after 10 days of infection the pathological features match in vivo infection. Thus, this model can be used to research mechanisms involving other dermatophytes.

The cause of dermatophytosis is exogenous sources not part of normal flora in healthy human or animal. Initial contact between arthroconidia and stratum corneum is an important moment within the process of initiation and skin infection. Dermatophyte attachment expressed carbohydrate specialized adhesive on the surface of microconidia which recognizes mannose and 
galactose. Adhesin may play an important role in attachment during the infection process [26, 27].

The attachment ability of fungi on host tissue is an important step of infection and the requirement of dermatophyte infection. Intradermal macroconidia inoculation in this research is very effective in improving direct infection right to invasion phase on the skin where this model confirmed epidermal differentiation process and skin permeability causing primary lesion within 2 hours and elevated up to 6 hours p.1. Dermatophytosis lesion appeared one-hour p.i. This result showed faster reaction compared to other researches. Intradermal infection may minimize similar infections caused by other microbes around the inoculation area. Intradermal infection is more efficient in causing dermatophytosis. In gross anatomy, the lesion produced by both M. gypseum and $M$. canis showed similar characteristics. This matches the statement from [12] which stated that in vitro test by stratum corneum biopsy later with keratinocyte being isolated showed maximum ability from Trichophyton $s p$. Macroconidia to induce attachment in human keratinocyte within 3-4 hours. [28] stated that T. mentagrosphytes attachment established with the fastest time of 6 hours after contact and germination occurred 4 hours postexposure. The attachment of T. mentagrosphytes was observed for 6 hours post-inoculation and the hyphae formed could be identified after 10 hours later. Experiment from [11] utilized the ex vivo model using human skin epidermis explant. In this model, the maximum attachability appeared.

\section{Conclusion}

Macroconidia is a component of the source of infection whose role is to induce a model of dermatophytosis and the severity of the primary injection is correlated with the duration and scale of improved clinical diagnosis. Lesions translated by M. gypseum invasive and M. canis do not have significant differences. This is a promising model for further research on research involved in the infection of both dermatophytes (M. gypseum and M. canis).

\section{Acknowledgment}

This research was supported by the 2019 Doctoral Research Grant, Ministry of Research, Technology and Higher Education, Republic of Indonesia.

\section{References}

1. Achterman RR, White TC: A foot in the door for dermatophyte research. PLoS Pathog. 2012;8(3):e1002564.

2. Fehr M.: Zoonotic Potential of Dermatophytosis in Small Mammals. Journal of Exotic Pet Medicine. 2015;24(3):308-16.

3. Cafarchia C, Weigl S, Figueredo LA, et al:: Molecular identification and phylogenesis of dermatophytes isolated from rabbit farms and rabbit farm workers. Veterinary Microbiology. 2012;154(3-4):395-402.
4. Tzar M, Zetti Z, Ramliza R, et al.: M. Dermatomycoses in Kuala Lumpur, Malaysia. Sains Malaysiana. 2014;43(11):1737-42.

5. Adzima V, Jamin F, Abrar M.: Isolasi dan identifikasi kapang penyebab Dermatofitosis pada Anjing di Kecamatan Syiah Kuala Banda Aceh. Journal Media Veterinarian. 2013;7(1):46-8.

6. Solank R.: Impurity profiling of active pharmaceutical ingredients and finished drug products. International journal of drug research and technology. 2017;2(3):7.

7. Putriningsih P, Arjentinia I.: Macroconidia of dermatophytes fungi on direct microscopic examinations. Journal of Veterinary Medicine and Animal Science. 2017;1(1):40-2.

8. Gräser $\mathrm{Y}$, Scott J, Summerbell R: The new species concept in dermatophytes-a polyphasic approach. Mycopathologia. 2008;166(5-6):239.

9. Shimamura T, Kubota N, Shibuya K.: Animal model of dermatophytosis. BioMed Research International. 2012;2012.

10. McGonigle P, Ruggeri B : Animal models of human disease: challenges in enabling translation. Biochemical pharmacology. 2014;87(1):162-71.

11. Kaufman G, Horwitz BA, Duek L, et al.:Infection stages of the dermatophyte pathogen Trichophyton: microscopic characterization and proteolytic enzymes. Medical Mycology. 2007;45(2):149-55.

12. Zurita J, Hay RJ: Adherence of Dermatophyte Microconidia and Arthroconidia to Human Keratinocytes In Vitro. Journal of Investigative Dermatology. 1987;89(5):529-34.

13. Aljabre S, Richardson M, Scott E, et al.: Adherence of arthroconidia and germlings of anthropophilic and zoophilic varieties of Trichophyton mentagrophytes to human corneocytes as an early event in the pathogenesis of dermatophytosis. Clinical and experimental dermatology. 1993;18(3):231-5.

14. Niimi K, Niimi M, Harada K, et al.: Macroconidial development and germination in Trichophyton mentagrophytes. Journal of investigative dermatology. 1988;90(2):165-9.

15. Yue X, Wang A, Li Q: The role of scanning electron microscopy in the direct diagnosis of onychomycosis. Scanning. 2018;2018.

16. Goldstein JI, Newbury DE, Michael JR, et al.: Scanning electron microscopy and X-ray microanalysis: Springer. 2017.

17. Cambier L, Mathy A, Baldo A, et al.: Feline polymorphonuclear neutrophils produce proinflammatory cytokines following exposure to Microsporum canis. Veterinary Microbiology. 2013;162(2-4):800-5.

18. Pihet M, Clément N, Kauffmann L, et al.: Diagnosis of dermatophytosis: an evaluation of direct examination using MycetColor ${ }^{\circledR}$ and MycetFluo ${ }^{\circledR}$. Diagnostic Microbiology and Infectious Disease. 2015;83(2):170-4.

19. Jensen RH, Arendrup MC: Molecular diagnosis of dermatophyte infections. Current opinion in infectious diseases. 2012;25(2):126-34.

20. Flower RJ, Gavins F, Halobetasol, et al.: The Comprehensive Pharmacology Reference. New York: Elsevier; 2008. p. 1-4. 
21. Hay RJ, Baran R.: Onychomycosis: A proposed revision of the clinical classification. Journal of the American Academy of Dermatology. 2011;65(6):1219-27.

22. Shenoy M, Teerthanath S, Kamaker VK, et al.: Comparison of potassium hydroxide mount and mycological culture with histopathologic examination using periodic acid-Schiff staining of the nail clippings in the diagnosis of onychomycosis. Indian journal of dermatology venereology and leprology. 2008;74(3):2269.

23. Shenoy M, Teerthanath S, Kamaker VK, et al.: Comparison of potassium hydroxide mount and mycological culture with histopathologic examination using periodic acid-Schiff staining of the nail clippings in the diagnosis of onychomycosis. Indian journal of dermatology venereology and leprology. 2008;74(3):2269.

24. Degreef H: Clinical forms of dermatophytosis (ringworm infection). Mycopathologia. 2008;166(56):257.

25. Liang PP, Huang XZ, Yi JL, et al.: A Trichophyton Rubrum Infection Model Based on the Reconstructed Human Epidermis-Episkin ${ }^{\circledR}$. Chinese medical journal. 2016;129(1):54.
26. Esquenazi D, De Souza W, Alviano CS, et al.: The role of surface carbohydrates on the interaction of microconidia of Trichophyton mentagrophytes with epithelial cells. FEMS Immunology \& Medical Microbiology. 2003;35(2):113-23.

27. Esquenazi D, Alviano CS, de Souza W, et al.: The influence of surface carbohydrates during in vitro infection of mammalian cells by the dermatophyte Trichophyton rubrum. Research in microbiology. 2004;155(3):144-53.

28. Aljabre SHM, Randhawa MA, Akhtar $\mathrm{N}$, et al.: Antidermatophyte activity of ether extract of Nigella sativa and its active principle, thymoquinone. Journal of Ethnopharmacology. 2005;101(1-3):116-9.

29. Tabart J, Baldo A, Vermout S, et al.: Reconstructed interfollicular feline epidermis as a model for Microsporum canis dermatophytosis. Journal of medical microbiology. 2007;56(7):971-5. 